PROCEEDINGS OF THE

AMERICAN MATHEMATICAL SOCIETY

Volume 136, Number 1, January 2008, Pages 359-365

S 0002-9939(07)09018-1

Article electronically published on September 7, 2007

\title{
UNIFORM PERIODIC POINT GROWTH IN ENTROPY RANK ONE
}

\author{
RICHARD MILES AND THOMAS WARD
}

(Communicated by Jane M. Hawkins)

\begin{abstract}
We show that algebraic dynamical systems with entropy rank one have uniformly exponentially many periodic points in all directions.
\end{abstract}

\section{INTRODUCTION}

Let $\alpha$ be an action of $\mathbb{Z}^{d}$ by continuous automorphisms of a compact metrizable abelian group $X$ (such a system is called an algebraic $\mathbb{Z}^{d}$-action). For a continuous map $\beta: X \rightarrow X$ write $h(\beta)$ for the topological entropy and

$$
\mathrm{F}(\beta)=\{x \in X \mid \beta x=x\}
$$

for the set of fixed points. The action $\alpha$ is said to have entropy rank one if, for each $\mathbf{n} \in \mathbb{Z}^{d}, h\left(\alpha^{\mathbf{n}}\right)<\infty$.

If $\alpha$ is a mixing entropy rank one action and the topological dimension $\operatorname{dim}(X)$ is finite, $\mathrm{F}\left(\alpha^{\mathbf{n}}\right)$ is finite for all $\mathbf{n} \in \mathbb{Z}^{d}$. Our purpose here is to show that under natural conditions $\left|\mathrm{F}\left(\alpha^{\mathbf{n}}\right)\right|$ exhibits uniform exponential growth. Write $\mathbf{n}_{j} \rightarrow \infty$ for a sequence in $\mathbb{Z}^{d}$ if for any finite set $F \subset \mathbb{Z}^{d}$ there is some $J$ for which $j>J$ implies that $\mathbf{n}_{j} \notin F$. Equivalently, this means $\left\|\mathbf{n}_{j}\right\| \rightarrow \infty$ as $j \rightarrow \infty$ where $\|\cdot\|$ is the Euclidean norm on $\mathbb{Z}^{d}$. The Noetherian condition mentioned in Theorem 1.1 is explained in Section 2

Theorem 1.1. Let $\alpha$ be a mixing algebraic $\mathbb{Z}^{d}$-action with entropy rank one on a finite-dimensional group $X$. Then there exist constants $C_{1}, C_{2} \geq 0$ such that

$$
\limsup _{\mathbf{n} \rightarrow \infty} \frac{1}{\|\mathbf{n}\|} \log \left|\mathrm{F}\left(\alpha^{\mathbf{n}}\right)\right|=C_{1}<\infty
$$

and

$$
\liminf _{\mathbf{n} \rightarrow \infty} \frac{1}{\|\mathbf{n}\|} \log \left|\mathrm{F}\left(\alpha^{\mathbf{n}}\right)\right|=C_{2} .
$$

If $\operatorname{dim}(X)>0$ and the action is Noetherian, then $C_{2}>0$.

An affirmative answer to Lehmer's problem (see Remark 2.3) would render the finite-dimension assumption in Theorem 1.1 redundant. The assumption is also not required for a mixing Noetherian entropy rank one algebraic action; in that setting

Received by the editors September 25, 2006.

2000 Mathematics Subject Classification. Primary 22D40, 37A15, 37A35.

This research was supported by E.P.S.R.C. grant EP/C015754/1. Both authors express their thanks to Graham Everest and Shaun Stevens for helpful discussions.

(C)2007 American Mathematical Society Reverts to public domain 28 years from publication 
it is a consequence of the Noetherian condition. Essentially, the only non-trivial conclusion in the theorem is that $C_{2}>0$.

The action $\alpha$ is expansive if there exists a neighbourhood $U$ of $0_{X}$ such that

$$
\bigcap_{\mathbf{n} \in \mathbb{Z}^{d}} \alpha^{\mathbf{n}}(U)=\left\{0_{X}\right\} .
$$

For $d=1$ and $\alpha$ expansive, the growth rate of periodic points exists, so $C_{1}=C_{2}$ in Theorem 1.1. The constant coincides with the entropy and is non-zero provided $X$ is infinite.

The $\mathbb{Z}^{2}$-action on a zero-dimensional group in Example 1.3 is expansive yet has $C_{2}=0$. Expansive actions on connected groups are more indicative of the import of a positive value for $C_{2}$. In terms of expansive subdynamics (see 3 . and [6]), there are sequences $\mathbf{n} \rightarrow \infty$ converging to non-expansive lines; along such sequences $\left|\mathrm{F}\left(\alpha^{\mathbf{n}}\right)\right|$ is much smaller than the same expression with $\mathbf{n}$ of similar Euclidean size and far from non-expansive directions. Nonetheless, there is a uniform exponential growth in all directions.

Example 1.2. Consider the $\mathbb{Z}^{2}$-action $\alpha$ dual to the $\mathbb{Z}^{2}$-action generated by the commuting maps $r \mapsto 2 r$ and $r \mapsto 3 r$ on $\mathbb{Z}\left[\frac{1}{6}\right]$. Table 1 shows the map

$$
\mathbf{n} \mapsto\left|\mathrm{F}\left(\alpha^{\mathbf{n}}\right)\right| \in \mathbb{N} \text {. }
$$

Notice that $\left|\mathrm{F}\left(\alpha^{-\mathbf{n}}\right)\right|=\left|\mathrm{F}\left(\alpha^{\mathbf{n}}\right)\right|$, so only the region $n_{2} \geq 0$ is shown, with $\infty$ denoting the lattice point $(0,0)$ corresponding to the identity map $\alpha^{(0,0)}$. In an expansive direction such as $(1,1)$ we have $\left|\mathrm{F}\left(\alpha^{n(1,1)}\right)\right|=6^{n}-1$ and the exponential growth rate along the sequence in italics is clear. For lattice points close to the non-expansive line $2^{x} 3^{y}=1$ we find (for example) that $\left|\mathrm{F}\left(\alpha^{(-5,3)}\right)\right|=5$, and it is not immediately clear that there is exponential growth along the sequence shown in bold; Theorem 1.1 asserts that there is.

Example 1.3. Consider the expansive $\mathbb{Z}^{2}$-action $\alpha$ on $X$ dual to the $\mathbb{Z}^{2}$-action generated by the commuting maps $r \mapsto u_{1} r$ and $r \mapsto u_{2} r$ on

$$
\mathbb{Z}\left[u_{1}^{ \pm 1}, u_{2}^{ \pm 1}\right] /\left\langle 2,1+u_{1}+u_{2}\right\rangle
$$

(this is an example of the type introduced by Ledrappier [12]; $X$ is a zero-dimensional group). As shown in [4], $\left|\mathrm{F}\left(\alpha^{(n, 0)}\right)\right|=2^{n-2^{\mathrm{ord}_{2}(n)}}$. In particular,

$$
\lim _{n \rightarrow \infty} \frac{1}{\left\|\left(2^{n}, 0\right)\right\|} \log \left|\mathrm{F}\left(\alpha^{\left(2^{n}, 0\right)}\right)\right|=0,
$$

showing that some assumption on the topological dimension of $X$ is needed to have $C_{2}>0$ in Theorem [1.1. If the map dual to $r \mapsto u_{2} r$ is ignored, then this

TABLE 1. Periodic point counts for $\times 2, \times 3$.

\begin{tabular}{ccccccccccc}
\hline 211 & 227 & 235 & 239 & 241 & 121 & 485 & 971 & 1943 & 3887 & 7775 \\
49 & 65 & 73 & 77 & 79 & 5 & 161 & 323 & 647 & 1295 & 2591 \\
$\mathbf{5}$ & 11 & 19 & 23 & 25 & 13 & 53 & 107 & 215 & 431 & 863 \\
23 & $\mathbf{7}$ & $\mathbf{1}$ & 5 & 7 & 1 & 17 & 35 & 71 & 143 & 287 \\
29 & 13 & $\mathbf{5}$ & 1 & 1 & 1 & 5 & 11 & 23 & 47 & 95 \\
31 & 5 & 7 & $\mathbf{1}$ & $\mathbf{1}$ & $\infty$ & 1 & 1 & 7 & 5 & 31 \\
\hline
\end{tabular}


example is a non-expansive (and non-Noetherian) $\mathbb{Z}$-action on a zero-dimensional group with $C_{2}=0$.

Example 1.4. The Noetherian condition is needed to have $C_{2}>0$ even for $d=1$ on connected groups. For example, the automorphism $\alpha$ dual to the map $r \mapsto 2 r$ on $\mathbb{Q}$ has $\left|\mathrm{F}\left(\alpha^{n}\right)\right|=1$ for all $n$. For rings between $\mathbb{Z}$ and $\mathbb{Q}$ a variety of exotic periodic point behavior is possible (see [20] or [21] for the details).

The full statement of Theorem 1.1 does however apply to Noetherian nonexpansive systems. Thus, for example, the genuinely partially hyperbolic systems such as that described by Damjanović and Katok [5, Ex. 7.3] satisfy the hypotheses.

\section{Proof of Theorem 1.1}

The case $d=1$ is covered by [4] and [16, so we may assume $d \geq 2$. Algebraic $\mathbb{Z}^{d}$-actions have a convenient description in terms of commutative algebra due to Kitchens and Schmidt [11].

Let $R_{d}=\mathbb{Z}\left[u_{1}^{ \pm 1}, \ldots, u_{d}^{ \pm 1}\right]$ be the ring of Laurent polynomials in commuting variables $u_{1}, \ldots, u_{d}$ with integer coefficients. If $\alpha$ is an algebraic $\mathbb{Z}^{d}$-action of a compact abelian group $X$, the character group $M=\widehat{X}$ has the structure of a discrete countable $R_{d}$-module, obtained by first identifying the dual automorphism $\widehat{\alpha}^{\mathbf{n}}$ with multiplication by the monomial $u^{\mathbf{n}}=u_{1}^{n_{1}} \ldots u_{d}^{n_{d}}$, and then extending additively to multiplication by polynomials. Conversely, any countable $R_{d^{-}}$-module $M$ has an associated algebraic $\mathbb{Z}^{d}$-action obtained by dualizing the action induced by multiplying by monomials on $M$. The action $\alpha=\alpha_{M}$ is described as Noetherian if the module $M$ is Noetherian. A full account of this correspondence and the resulting theory is given in Schmidt's monograph [19.

Entropy rank one actions are described in [7] and developments concerning their periodic points may be found in the papers [16] and [18]. By a slight abuse of notation, write $h(\cdot)$ for the topological entropy of maps and for the extension of the entropy function to all of $\mathbb{R}^{d}$ in the sense explained below.

Proposition 2.1. Let $\alpha_{M}$ be a mixing algebraic $\mathbb{Z}^{d}$-action with entropy rank one on a finite-dimensional group. Then

(1) The set of associated primes of the associated $R_{d}$-module, $\operatorname{Asc}(M)$, is finite. For each $\mathfrak{p} \in \operatorname{Asc}(M)$, the domain $R_{d} / \mathfrak{p}$ has Krull dimension 1 and its field of fractions $\mathbb{K}(\mathfrak{p})$ is a global field.

(2) There exist positive integers $m(\mathfrak{p}), \mathfrak{p} \in \operatorname{Asc}(M)$, such that for every nonzero $\mathbf{n} \in \mathbb{Z}^{d}$,

$$
h\left(\alpha_{M}^{\mathbf{n}}\right)=\sum_{\mathfrak{p} \in \operatorname{Asc}(M)} m(\mathfrak{p}) h\left(\alpha_{R_{d} / \mathfrak{p}}^{\mathbf{n}}\right)
$$

and

$$
\left|\mathrm{F}\left(\alpha_{M}^{\mathbf{n}}\right)\right| \leqslant \prod_{\mathfrak{p} \in \operatorname{Asc}(M)}\left|\mathrm{F}\left(\alpha_{R_{d} / \mathfrak{p}}^{\mathbf{n}}\right)\right|^{m(\mathfrak{p})}
$$

with equality if $M$ is Noetherian.

(3) For each $\mathfrak{p} \in \operatorname{Asc}(M)$, there is a finite set of places $\mathcal{S}(\mathfrak{p})$ of $\mathbb{K}(\mathfrak{p})$ such that

$$
h\left(\alpha_{R_{d} / \mathfrak{p}}^{\mathbf{n}}\right)=\sum_{v \in \mathcal{S}(\mathfrak{p})} \max \left\{\mathbf{l}_{v} \cdot \mathbf{n}, 0\right\}>0
$$


and

$$
\left|\mathrm{F}\left(\alpha_{R_{d} / \mathfrak{p}}^{\mathbf{n}}\right)\right|=\prod_{v \in \mathcal{S}(\mathfrak{p})}\left|\xi_{\mathfrak{p}}^{\mathbf{n}}-1\right|_{v},
$$

where $\xi_{\mathfrak{p}}=\left(\bar{u}_{1}, \ldots, \bar{u}_{d}\right)$ denotes the image of $u$ in $\left(R_{d} / \mathfrak{p}\right)^{d}$ and $\mathbf{l}_{v}=$ $\left(\log \left|\bar{u}_{1}\right|_{v}, \ldots, \log \left|\bar{u}_{d}\right|_{v}\right)$.

Proof. Let $\mathbf{n} \in \mathbb{Z}^{d}$ be non-zero. Since $\alpha_{M}$ is mixing, [19, Prop. 6.6] shows that for each $\mathfrak{p} \in \operatorname{Asc}(M), \alpha_{R_{d} / \mathfrak{p}}^{\mathbf{n}}$ is ergodic, so $h\left(\alpha_{R_{d} / \mathfrak{p}}^{\mathbf{n}}\right)>0$. It follows from [7] that for each $\mathfrak{p} \in \operatorname{Asc}(M), R_{d} / \mathfrak{p}$ has Krull dimension 1 and $\mathbb{K}(\mathfrak{p})$ is a global field. If $\operatorname{char}\left(R_{d} / \mathfrak{p}\right)>0$, then $h\left(\alpha_{R_{d} / \mathfrak{p}}^{\mathbf{n}}\right) \geq \log 2$. Via Yuzvinski1's formula (see [19, Th. 14.1] or [23]), this implies there are only finitely many such $\mathfrak{p} \in \operatorname{Asc}(M)$, as $h\left(\alpha_{R_{d} / \mathfrak{p}}^{\mathbf{n}}\right)<\infty$.

Also, there can be only finitely many $\mathfrak{p} \in \operatorname{Asc}(M)$ with $\operatorname{char}\left(R_{d} / \mathfrak{p}\right)=0$, as

$$
\operatorname{dim}(X) \geqslant\left|\left\{\mathfrak{p} \in \operatorname{Asc}(M): \operatorname{char}\left(R_{d} / \mathfrak{p}\right)=0\right\}\right| .
$$

This establishes (1).

The method of [7, Lem. 8.2] shows that in any prime filtration of a Noetherian submodule of $M$, each prime $\mathfrak{p} \in \operatorname{Asc}(M)$ appears with a maximum multiplicity

$$
m(\mathfrak{p})=\operatorname{dim}_{\mathbb{K}(\mathfrak{p})}\left(M \otimes_{R_{d}} \mathbb{K}(\mathfrak{p})\right),
$$

which is finite by similar reasoning to the proof of (1). By adopting an algorithm for obtaining a prime filtration that selects the associated primes of $M$ first, one obtains a Noetherian submodule $L \subset M$ such that each prime $\mathfrak{p} \in \operatorname{Asc}(M)$ appears with multiplicity $m(\mathfrak{p})$ in a filtration of $L$. Furthermore, if $L \neq M$, then each $\mathfrak{q} \in$ $\operatorname{Asc}(M / L)$ is maximal and $R_{d} / \mathfrak{q}$ is a finite field. Hence, Yuzvinskiì's formula shows that $h\left(\alpha_{M / L}^{\mathbf{n}}\right)=0$ and $h\left(\alpha_{M}^{\mathbf{n}}\right)=h\left(\alpha_{L}^{\mathbf{n}}\right)$; the formula (2.1) then follows from [15, Lem. 4.3].

If $M$ is Noetherian, equality in (2.2) is given by [16, Th. 3.2]. If $M$ is not Noetherian, (2.2) follows from [16, Th. 3.2] applied to $L$, together with the inequality

$$
\left|\mathrm{F}\left(\alpha_{M}^{\mathbf{n}}\right)\right| \leqslant\left|\mathrm{F}\left(\alpha_{L}^{\mathbf{n}}\right)\right|
$$

which is established using a similar method to the proof of [17, Lem. 2.6].

Finally, the entropy formula (2.3) follows from [7, Prop. 8.5] and the periodic point counting formula (2.4) is [16, Lem 3.1].

Proof of Theorem 1.1, Let $M=\widehat{X}$ denote the dual $R_{d}$-module and let $\mathfrak{p} \in \operatorname{Asc}(M)$. For a fixed $\mathfrak{p} \in \operatorname{Asc}(M)$ and any non-zero $\mathbf{n} \in \mathbb{Z}^{d}$, set

$$
f(\mathbf{n})=\frac{1}{\|\mathbf{n}\|} \log \left|\mathrm{F}\left(\alpha_{R_{d} / \mathfrak{p}}^{\mathbf{n}}\right)\right| .
$$

Let $h: \mathbb{R}^{d} \rightarrow \mathbb{R}_{\geqslant 0}$ denote the directional entropy function for the action $\alpha_{R_{d} / \mathfrak{p}}$. This is the function obtained by extending the entropy formula (2.3) to values of $\mathbb{R}^{d}$ (see [7, Sec. 8] for further details).

For any vector $\mathbf{v} \in \mathbb{R}^{d} \backslash\{0\}$, write $\widehat{\mathbf{v}} \in \mathrm{S}_{d-1}$ for the unique vector of unit length with the property that $\mathbf{v}=\lambda \widehat{\mathbf{v}}$ for some scalar $\lambda>0$. From (2.3) and (2.4) it follows that

$$
f(\mathbf{n})=g(\mathbf{n})+h(\widehat{\mathbf{n}})
$$


where

$$
g(\mathbf{n})=\frac{1}{\|\mathbf{n}\|} \sum_{v \in \mathcal{S}(\mathfrak{p})} \log \left|1-\phi_{v}(\mathbf{n})\right|_{v}
$$

and

$$
\phi_{v}(\mathbf{n})= \begin{cases}\xi_{\mathfrak{p}}^{-\mathbf{n}} & \text { if }\left|\xi_{\mathfrak{p}}^{\mathbf{n}}\right|_{v}>1 \\ \xi_{\mathfrak{p}}^{\mathbf{n}} & \text { if }\left|\xi_{\mathfrak{p}}^{\mathbf{n}}\right|_{v} \leqslant 1\end{cases}
$$

Notice that $\phi_{v}(\mathbf{n}) \neq 1$ if $\mathbf{n} \neq 0$ by the assumption that the action is mixing.

To establish the lower bound $C_{2}>0$ in Theorem 1.1, first note that equality in (2.2) gives the expression

$$
\frac{1}{\|\mathbf{n}\|} \log \left|\mathrm{F}\left(\alpha_{M}^{\mathbf{n}}\right)\right|
$$

as a finite sum of terms of the form (2.5) and, crucially, the assumption $\operatorname{dim}(X)>0$ means at least one of these terms arises from a prime $\mathfrak{p}$ such that $\mathbb{K}(\mathfrak{p})$ is an algebraic number field (rather than a function field of positive characteristic). For the lower bound, it is therefore enough by (2.2) to consider only the asymptotic behavior of $f$ with the assumption that it arises from such a prime.

We need to know that $g$ does not make an asymptotic contribution. It is clear that $g$ cannot be too large, since

$$
\prod_{v \in \mathcal{S}(\mathfrak{p})}\left|1-\phi_{v}(\mathbf{n})\right|_{v} \leq 2^{|\mathcal{S}(\mathfrak{p})|} .
$$

On the other hand, it cannot be large and negative for the following reason. If $v$ is an infinite (Archimedean) place, then Baker's theorem [1] can be used to find constants $A, B \geq 0$ such that

$$
\left|1-\phi_{v}(\mathbf{n})\right|_{v} \geq \frac{A}{\max \left\{n_{i}\right\}^{B}}
$$

(see 4] or 9] for similar arguments; roughly speaking, the issue is to bound the proximity of $\xi_{\mathfrak{p}}^{\mathbf{n}}$ and 1 in terms of $\mathbf{n}$ ). If $v$ is a finite place, then Yu's $p$-adic bounds for linear forms in logarithms [22] give a similar lower bound (note that $d \geq 2$ by assumption). Since $\mathcal{S}(\mathfrak{p})$ is finite, this shows that

$$
g(\mathbf{n}) \rightarrow 0 \text { as } \mathbf{n} \rightarrow \infty .
$$

Assume, for a contradiction, that $C_{2}=0$. Then there is a sequence $\mathbf{n}_{j} \rightarrow \infty$ as $j \rightarrow \infty$ with the property that

$$
\lim _{j \rightarrow \infty} f\left(\mathbf{n}_{j}\right)=0 .
$$

It follows from (2.6) and (2.7) that

$$
h\left(\widehat{\mathbf{n}_{j}}\right) \rightarrow 0 \text { as } j \rightarrow \infty .
$$

Now the entropy function $h$ restricted to the unit sphere $S_{d-1}$ is a continuous function on a compact set, so (2.9) implies that there is some $\mathbf{v} \in \mathrm{S}_{d-1}$ for which

$$
h(\mathbf{v})=0 .
$$

If the ray through $\mathbf{v}$ happens to meet a point $\mathbf{m} \in \mathbb{Z}^{d}$, then we have an immediate contradiction: the automorphism $\alpha_{R_{d} / \mathfrak{p}}^{\mathbf{m}}$ would have zero entropy, contradicting (2.3). In order to show that any point $\mathbf{v}$ with (2.10) gives a contradiction we need to make a quantitative version of that argument. 
The explicit formula (2.3) for $h$ means there is a list of vectors

$$
\mathbf{a}_{1}, \ldots, \mathbf{a}_{r} \in \mathbb{R}^{d}
$$

with the property that for any $\mathbf{u} \in \mathbb{R}^{d}$,

$$
h(\mathbf{u})=\mathbf{u} \cdot \mathbf{a}_{k},
$$

for some $k, 1 \leqslant k \leqslant r$. It follows that for any $\mathbf{n} \in \mathbb{Z}^{d}$ and $\mathbf{u} \in \mathbb{R}^{d}$,

$$
|h(\mathbf{n})-h(\mathbf{u})| \leqslant \max _{1 \leqslant k \leqslant r} \max _{1 \leqslant i \leqslant d}\left\{\left|a_{k, i}\right|\right\}\|\mathbf{n}-\mathbf{u}\|,
$$

where $\mathbf{a}_{k}=\left(a_{k, 1}, \ldots, a_{k, d}\right)$. In particular, since $h(\lambda \mathbf{v})=0$ for all $\lambda>0$ we may find a sequence $\left(\mathbf{m}_{j}\right)$ of vectors in $\mathbb{Z}^{d}$ with $\left\|\mathbf{m}_{j}-\lambda_{j} \mathbf{v}\right\| \rightarrow 0$ as $j \rightarrow \infty$ (for some sequence $\left(\lambda_{j}\right)$ of scalars) and hence have

$$
h\left(\mathbf{m}_{j}\right) \rightarrow 0 \text { as } j \rightarrow \infty .
$$

On the other hand, the dual group of $R_{d} / \mathfrak{p}$ is connected and finite-dimensional. Hence, by Yuzvinskiı̌'s formula, for any $\mathbf{n} \neq 0$,

$$
h(\mathbf{n}) \geqslant \mathrm{m}(P)>0
$$

where $\mathrm{m}(P)=\int_{0}^{1} \log \left|P\left(e^{2 \pi \mathrm{i} s}\right)\right| \mathrm{d} s$ denotes the logarithmic Mahler measure of some polynomial $P$ of degree no greater than $\operatorname{dim}_{\mathbb{Q}}(\mathbb{K}(\mathfrak{p}))$. It follows that there is a constant $C>0$ (depending only on $\mathfrak{p}$ ) for which

$$
h(\mathbf{n})>C>0 \text { for any } \mathbf{n} \neq 0
$$

(the existence of a lower bound for the non-zero logarithmic Mahler measure of polynomials of bounded degree is well known; see [2] or 8 for the background). This lower bound contradicts (2.11), so (2.8) is impossible. Thus $C_{2}>0$.

The upper bound is clear: the inequality (2.2) together with the explicit formula (2.4) gives a uniform constant $C_{1}$ with the property that

$$
\left|\mathrm{F}\left(\alpha^{\mathbf{n}}\right)\right| \leq C_{1}^{\max _{1 \leqslant i \leqslant d}\left\{n_{i}\right\}} .
$$

Remark 2.2. Baker's theorem provides the key estimates in many dynamical problems; see [4] and 9] for examples. As pointed out by Lind 14], in order to establish the logarithmic growth rate of periodic points for a quasihyperbolic toral automorphism (a typical application), sometimes all that is needed is a weaker and earlier result due to Gel'fond [10. Here we need something closer to the full weight of the theorems of Baker and $\mathrm{Yu}$, because we are in a higher rank.

Remark 2.3. Lehmer's problem 13 asks if there is a uniform lower bound for all positive Mahler measures. As shown by Lind (see [15]) this is equivalent to a uniform lower bound for the topological entropy of any mixing compact group automorphism. If 'Lehmer's conjecture' (that there is such a bound and that it is attained by the expected polynomial) holds, then the topological entropy of any compact group automorphism with positive entropy is at least

$$
0.162 \cdots=\mathrm{m}\left(x^{10}+x^{9}-x^{7}-x^{6}-x^{5}-x^{4}-x^{3}+x+1\right) .
$$

This does not imply a uniform bound for $C_{2}$ in Theorem 1.1 because $C_{2}$ is influenced by the geometry of the acting group as well as the collection of maps in its image. For example, the $\mathbb{Z}^{2}$-action $\alpha$ corresponding to the module $R_{2} /\left\langle u_{1}-2, u_{1}^{k} u_{2}-3\right\rangle$ has the property that $h\left(\alpha^{(k, 1)}\right)=\log 3$, so the corresponding constant $C_{2}$ cannot exceed $\log 3 / \sqrt{1+k^{2}}$. 


\section{REFERENCES}

[1] A. Baker. Transcendental number theory, Cambridge University Press, London, 1975. MR 0422171 (54:10163)

[2] P. E. Blanksby and H. L. Montgomery, Algebraic integers near the unit circle, Acta Arith., 18 (1971), 355-369. MR0296021 (45:5082)

[3] Mike Boyle and Douglas Lind, Expansive subdynamics, Trans. Amer. Math. Soc. 349 (1997), no. 1, 55-102. MR1355295 (97d:58115)

[4] Vijay Chothi, Graham Everest, and Thomas Ward, $S$-integer dynamical systems: Periodic points, J. Reine Angew. Math. 489 (1997), 99-132. MR.1461206 (99b:11089)

[5] Danijela Damjanović and Anatole Katok, Local rigidity of partially hyperbolic actions of $\mathbb{Z}^{k}$ and $\mathbb{R}^{k}, k \geq 2$. I. KAM method and actions on the torus, Preprint.

[6] Manfred Einsiedler, Douglas Lind, Richard Miles, and Thomas Ward, Expansive subdynamics for algebraic $\mathbb{Z}^{d}$-actions, Ergodic Theory Dynam. Systems 21 (2001), no. 6, 1695-1729. MR.1869066 (2004c:37033)

[7] Manfred Einsiedler and Douglas Lind, Algebraic $\mathbb{Z}^{d}$-actions of entropy rank one, Trans. Amer. Math. Soc. 356 (2004), no. 5, 1799-1831. MR2031042 (2005a:37009)

[8] Graham Everest and Thomas Ward, Heights of polynomials and entropy in algebraic dynamics, Springer-Verlag London, Ltd., London, 1999. MR.1700272 (2000e:11087)

[9] Graham Everest, Richard Miles, Shaun Stevens and Thomas Ward, Orbit-counting in nonhyperbolic dynamical systems, J. Reine Angew. Math., 608 (2007), 155-182.

[10] A. O. Gel'fond, Transcendental and algebraic numbers, Dover Publications, Inc., New York 1960. Translated from the first Russian edition by Leo F. Boron. MR0111736 (22:2598)

[11] Bruce Kitchens and Klaus Schmidt, Automorphisms of compact groups, Ergodic Theory Dynam. Systems 9 (1989), no. 4, 691-735. MR1036904 (91g:22008)

[12] François Ledrappier, Un champ markovien peut être d'entropie nulle et mélangeant, $C . R$. Acad. Sci. Paris Sér. A-B 287 (1978), no. 7, A561-A563. MR0512106 (80b:28030)

[13] D. H. Lehmer, Factorization of certain cyclotomic functions, Ann. of Math. (2) 34 (1933), no. 3, 461-479. MR 1503118

[14] Douglas Lind, Dynamical properties of quasihyperbolic toral automorphisms, Ergodic Theory Dynam. Systems 2 (1982), no. 1, 49-68. MR684244 (84g:28017)

[15] Douglas Lind, Klaus Schmidt, and Thomas Ward, Mahler measure and entropy for commuting automorphisms of compact groups, Invent. Math. 101 (1990), no. 3, 593-629. MR:1062797 $(92 \mathrm{j}: 22013)$

[16] Richard Miles, Zeta functions for elements of entropy rank one actions, Ergodic Theory Dynam. Systems, 27 (2007), no. 2, 567-582. MR2308145

[17] Richard Miles, Periodic points of endomorphisms on solenoids and related groups, preprint, 2006.

[18] Richard Miles and Thomas Ward, Periodic point data detects subdynamics in entropy rank one, Ergodic Theory Dynam. Systems 26 (2006), no. 6, 1913-1930. MR2279271

[19] Klaus Schmidt, Dynamical systems of algebraic origin, Progress in Mathematics, vol. 128, Birkhäuser Verlag, Basel, 1995. MR.1345152 (97c:28041)

[20] Thomas Ward. An uncountable family of group automorphisms, and a typical member. Bull. London Math. Soc. 29 (1997) no. 5, 577-584. MR1458718 (98k:22028)

[21] Thomas Ward. Almost all $S$-integer dynamical systems have many periodic points. Ergodic Theory Dynam. Systems, 18 (1998), no. 2, 471-486. MR.1619569 (99k:58152)

[22] Kunrui Yu. Linear forms in p-adic logarithms. II. Compositio Math., 74 (1990), no. 1, 15-113. MR:1055245(91h:11065a)

[23] S. A. Yuzvinskiǔ, Calculation of the entropy of a group-endomorphism, Sibirsk. Mat. Z̆., 8, (1967), 230-239. MR0214726 (35:5575)

School of Mathematics, KTH, SE-100 44 Stockholm, Sweden

School of Mathematics, University of East Anglia, Norwich, NR4 7TJ, United KingDOM 